\title{
Propuesta de un nuevo índice de calidad global de la alimentación
}

\author{
A proposed new index of \\ global food quality
}

\begin{abstract}
Various methodologies have been proposed to assess the overall quality of diet, but there are no specific indicators in Latin America. The purpose of this work was to propose a Healthy Eating Index according to the recommendations of the Chilean Ministry of Health and to validate their application in a national sample of students. A food frequency survey with 12 variables was used: 5 healthy foods, 4 unhealthy foods and 3 main meals. Each variable was rated from 1 (less healthy) to 10 (recommended by the Ministry of Health). Total scores were used to classify diets as healthy (90-120), in need of changes (60-89) and unhealthy $(<60)$. The questionnaire was administered to 9,452 university students and was evaluated according to sex, age and nutritional status. The median score was 65.5 (54.5 to 77.9: p25-75) and 9.2\% had healthy diets, $55.3 \%$ needed changes and $35.5 \%$ had unhealthy diets, without differences by sex and modest differences by nutritional status $(p<0.05)$. Younger age related to an unhealthy $\operatorname{diet}(p<0.005)$. The instrument is easily applied by health professionals and can be adapted to the recommendations of each country. There are serious deficiencies in diet quality among college students, regardless of their nutritional status and sex. Key words: Index; feeding; healthy; students; Chile.
\end{abstract}

\section{INTRODUCCIÓN}

Los estilos de vida tienen una directa asociación con el riesgo de desarrollar las principales enfermedades crónicas no transmisibles, grave problema de salud pública, de incidencia creciente en todas las regiones del mundo. La relevancia de cada factor de riesgo varía entre los países, pero entre ellos destaca el tabaquismo, consumo de alcohol, sedentarismo y la calidad de la alimentación, además de algunos factores genéticos. La alimentación puede actuar como un factor protector o de riesgo en relación a las enfermedades cardiovasculares, diferentes tipos de cáncer, diabetes, osteoporosis, entre varias otras patologías (1-4).

Para analizar la alimentación tradicionalmente se han utilizado encuestas alimentarias, ya sea de tendencia de consumo o de recordatorio de 24 horas. A partir de ellas se evalúa el consumo de cada alimento, grupos de alimentos y de nutrientes y su grado de adecuación a las recomendaciones de ingesta (5-8). Aunque las encuestas representan un instrumento muy valioso para evaluar el consumo deficiente o excesivo de nutrientes críticos, no permiten establecer un
Rinat Ratner (1)

Paulina Hernández (2)

Jorge Martel (2)

Eduardo Atalah (3) Chile.

(2) Sodexo, Servicios de beneficios e incentivos, Santiago, Chile. (3) Facultad de Medicina, Universidad de Chile, Santiago, Chile

Dirigir la correspondencia a: Profesora Rinat Ratner Goldenberg Directora Nutrición y Dietética
Facultad de Medicina, Universidad del Desarrollo-Clínica Alemana Email: rratner@udd.cl

Este trabajo fue recibido el 17 de Marzo de 2016 y aceptado para ser publicado el 13 de Diciembre de 2016.

diagnóstico global de la calidad de la alimentación a nivel individual o poblacional.

Para lograr un diagnóstico más integral sobre la alimentación, en los últimos 20 años se han definido diversos índices de calidad global de la dieta, construidos a partir de algoritmos que permiten categorizar cuan saludable es el patrón de alimentación de los individuos. Los índices propuestos se basan en los conocimientos actuales en nutrición y en las guías alimentarias de un determinado país y permiten identificar los principales factores de riesgo alimentario de las enfermedades crónicas no transmisibles. Existen numerosos índices, que se pueden clasificar en tres categorías principales: a) basados en nutrientes; b) basados en alimentos o grupos de alimentos; c) índices combinados. A estos últimos pertenecen la mayoría de los índices, los cuales consideran la adecuación de la dieta a las recomendaciones de consumo de los principales grupos de alimentos y de los macronutrientes (9-14).

El índice de alimentación saludable (15), el índice de calidad de la dieta (16), el indicador de dieta saludable (17) y el score de la dieta mediterránea (18), son los cuatro indicadores 
originales que se han referenciado y validado más extensamente. A partir de ellos diversos índices se han adaptado a las recomendaciones de un determinado país, incluyendo más recientemente otros que incluyen además del grado de adherencia a los hábitos de vida mediterránea (19).

Diversos estudios en Chile, así como las Encuestas Nacionales de Salud y de Consumo Alimentario muestran que una alta proporción de la población presenta un bajo consumo de frutas, verduras, lácteos, pescado y leguminosas e inversamente un consumo excesivo de alimentos no saludables con alto contenido de energía, grasas, azúcar y sodio (20-22).

Al menos dos estudios nacionales han evaluado la calidad de la alimentación en escolares y adultos adaptando la metodología propuesta por Kennedy y cols. (Healthy Eating Index), demostrando que una muy baja proporción de la población presenta una alimentación saludable y la gran mayoría necesita cambios importantes $(23,24)$. Aunque esta información es muy valiosa para definir y evaluar políticas públicas en relación a alimentación y nutrición, tiene la limitante de basarse en el registro de un día. Adicionalmente es complejo calcular el índice, porque se requiere el análisis de la ingesta de grasas totales, grasas saturadas, azúcar, sodio y energía (15).

El objetivo de este estudio es proponer y evaluar un Índice de Calidad de la Alimentación basado en las guías alimentarias del Ministerio de Salud en Chile, que sea fácil de aplicar por los diferentes profesionales de la salud.

\section{MATERIAL Y METODO}

Se diseñó una encuesta alimentaria de frecuencia de consumo con 12 variables, que incluía 5 grupos de alimentos saludables, 4 poco saludables y 3 sobre la frecuencia de las comidas. A partir de las primeras aplicaciones se realizaron reuniones de grupos focales con los encuestados, que permitieron algunos ajustes antes de establecer la versión definitiva, aunque no fue validada con una prueba de consistencia pro- piamente tal. Las variables estudiadas fueron la frecuencia de consumo diaria o semanal de: a) Frutas, verduras, pescados, leguminosas y leche o derivados; b) Frituras, bebidas con azúcar, pasteles/galletas/tortas/dulces y azúcar y c) Desayuno, almuerzo y cena.

Para cada una de estas variables se consideraron las siguientes alternativas: 2 o más veces al día, 1 vez al día, 4 a 6 veces a la semana, 2 a 3 veces a la semana, una vez a la semana y ocasional o nunca. En base a esta información se asignaron puntajes, siendo 10 el valor ideal según las guías alimentarias y el puntaje 1 lo menos saludable (tabla 1). El puntaje máximo con las 12 variables estudiadas es 120 , el que fue clasificado según el siguiente criterio: saludable: $90-120$ puntos; necesita cambios: 60-89 puntos; poco saludable: $<60$ puntos.

Entre los años 2010 y 2013 profesionales nutricionistas entrenadas aplicaron la encuesta a estudiantes universitarios de 54 universidades o institutos profesionales, en 11 regiones del país, previa aprobación del Comité de Ética de la Universidad del Desarrollo. Adicionalmente midieron el peso y la talla, siguiendo técnicas estandarizadas. El peso se obtuvo con ropa ligera y descalzo con balanza portátil marca SECA, con precisión de 100 gramos, calibrada periódicamente. La talla se midió con tallímetro portátil marca SECA con 0,1 cm de precisión. A partir de del peso y la talla se calculó el índice de masa corporal $\left(\mathrm{Kg} / \mathrm{m}^{2}\right)$ y se clasificó el estado nutricional según la norma OMS: enflaquecido $<18,5$; normo peso 18,5 24,9 ; sobrepeso $25,0-29,9$ y obeso $\geq 30$.

Los datos fueron analizados con el programa STATA11.0. Las variables de frecuencia de consumo no tenían una distribución normal de acuerdo al test de Shapiro Wilk, por lo que se analizó la mediana y percentiles 25 y 75 de cada una de ellas y se compararon las diferencias según sexo, por medio del test de Kruskal Wallis. La calidad global de la alimentación (variable categórica) se comparó en función del sexo, edad y estado nutricional a través del test $x 2$, considerando significativo $p<0,05$.

TABLA1

Puntaje asignado según frecuencia de consumo de alimentos saludables, no saludables y tiempos de comida.

\begin{tabular}{|c|c|c|c|c|c|c|}
\hline & $>2$ /día & 1 al día & 4-6/sem & $2-3 /$ sem & $1 /$ sem & $\begin{array}{c}\text { Ocasional } \\
\text { o nunca }\end{array}$ \\
\hline \multicolumn{7}{|l|}{ Alimentos saludables } \\
\hline Verduras & 10 & 7,5 & 5 & 2,5 & 1 & 1 \\
\hline Frutas & 10 & 7,5 & 5 & 2,5 & 1 & 1 \\
\hline Leche o derivados & 10 & 7,5 & 5 & 2,5 & 1 & 1 \\
\hline Legumbres & 10 & 10 & 10 & 10 & 7,5 & 1 \\
\hline Pescado & 10 & 10 & 10 & 10 & 7,5 & 1 \\
\hline \multicolumn{7}{|l|}{ Alimentos no saludables } \\
\hline Pasteles,galletas, dulces & 1 & 1 & 2,5 & 5 & 7,5 & 10 \\
\hline Bebidas con azúcar & 1 & 1 & 2,5 & 5 & 7,5 & 10 \\
\hline Azúcar & 1 & 1 & 2,5 & 5 & 7,5 & 10 \\
\hline Frituras & 1 & 1 & 2,5 & 5 & 7,5 & 10 \\
\hline \multicolumn{7}{|l|}{ Comidas } \\
\hline Desayuno & 5 & 10 & 5 & 2,5 & 1 & 1 \\
\hline Almuerzo & 5 & 10 & 5 & 2,5 & 1 & 1 \\
\hline Cena & 5 & 10 & 5 & 2,5 & 1 & 1 \\
\hline
\end{tabular}




\section{RESULTADOS}

Se estudiaron 9.460 estudiantes con un promedio de edad $21,3 \pm 2,5$ años (rango de 17 a 29 años) sin grandes diferencias según sexo. El análisis del estado nutricional mostró un predominio del peso normal, aunque el $27,2 \%$ presentada exceso de peso, con una situación más negativa en las mujeres (tabla 2).

La mediana del puntaje de cada indicador de la calidad de la alimentación y del índice total se presenta en la tabla 3. El índice global mostró un valor de 65,5 (p25-75: 54,5-77,9), que refleja un cumplimiento de un poco más de la mitad de las conductas saludables esperadas, sin diferencias por sexo. El puntaje más alto se obtuvo en la frecuencia de consumo del almuerzo y los más bajos por alta frecuencia de consumo de azúcar y baja frecuencia de la cena. En general los puntajes son más bajos en los alimentos no saludables, lo que reflejan un alto consumo de dulces/galletas, bebidas, azúcar y frituras. Existen algunas diferencias por sexo, especialmente por un mayor consumo de verduras, bebidas con azúcar y frituras en las mujeres.

Solo un 9,3\% de la muestra presentaba una alimentación saludable, un 55,3\% necesitaba cambios y un 35,4\% tenía alimentación poco saludable. No hubo diferencias por sexo (tabla 4). El análisis por las diferentes variables estudiadas mostró una situación levemente más desfavorable en las personas de bajo peso y obesas $(p<0,05)$, en los adolescentes $(p<0,005)$ y en la zona sur del país $(p<0,001)$.

Finalmente se analizó la calidad de la alimentación en los 4 años en que se aplicó la encuesta, observándose una tendencia positiva con un incremento de 5,5\% en la alimentación saludable y una reducción similar en la categoría no saludable (tabla 5).

\section{DISCUSIÓN}

El índice propuesto, en concordancia con los resultados de otros estudios nacionales, muestra que una muy baja fracción de la población cumple con las normas alimentarias

\section{TABLA 2}

Evaluación del estado nutricional según sexo.

\begin{tabular}{|c|c|c|c|c|c|c|}
\hline \multirow[t]{2}{*}{ Diagnóstico } & \multicolumn{2}{|c|}{ Mujer } & \multicolumn{2}{|c|}{ Hombre } & \multicolumn{2}{|c|}{ Total } \\
\hline & $\mathrm{n}$ & $\%$ & $\mathrm{n}$ & $\%$ & $\mathrm{n}$ & $\%$ \\
\hline Bajo peso & 250 & 4,6 & 58 & 1,5 & 308 & 3,3 \\
\hline Normal & 3.910 & 72,6 & 2.598 & 65,3 & 6.508 & 69,5 \\
\hline Sobre peso & 1.016 & 18,9 & 1.123 & 28,2 & 2.139 & 22,8 \\
\hline Obeso & 213 & 3,9 & 199 & 5,0 & 412 & 4,4 \\
\hline Total & 5.389 & 100,0 & 3.978 & 100,0 & 9.367 & 100,0 \\
\hline$x 2181,6 p<0,001$ & & & & & & \\
\hline
\end{tabular}

TABLA 3

Mediana de puntaje de cada variable del índice de calidad global de la dieta según sexo.

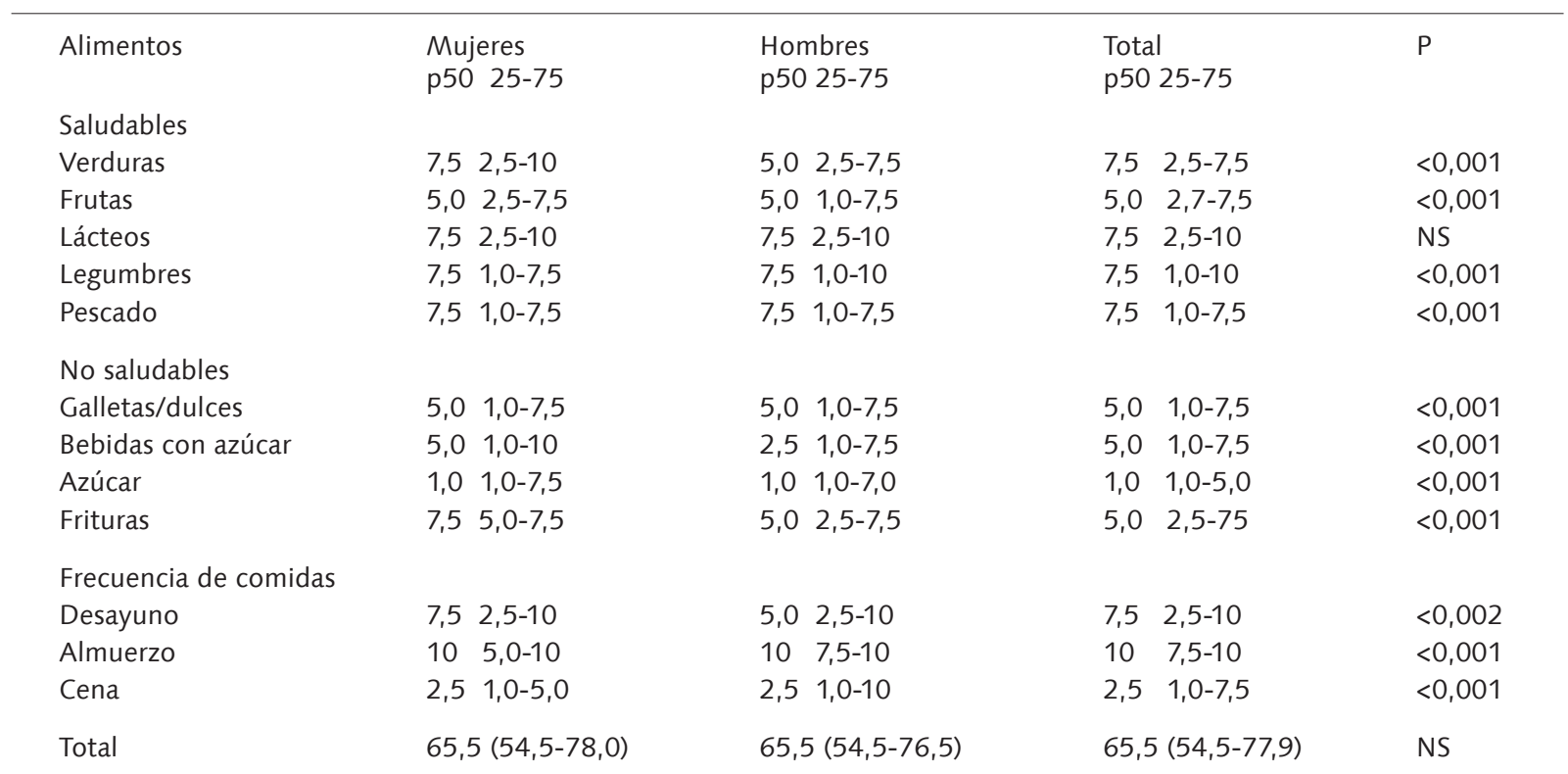


del Ministerio de Salud o tiene una alimentación saludable (20-24). El mismo hecho se observa en prácticamente todos los estudios realizados en relación a este tema, en países con diferentes grados de desarrollo. Así por ejemplo, un 80,2\% de los estudiantes universitarios en México tenían una alimentación no saludable, un 19,7\% necesitaba cambios y solo un $0,1 \%$ fue evaluado como saludable (25). Otro estudio en adultos mayores en Brasil determinó que el 74,2\% de la muestra necesitaba cambios en su alimentación y ninguno cumplía con una alimentación saludable (26).

La mediana de puntaje observada en el presente estudio fue de un 54\% del puntaje máximo, lo que también es consistente con lo observado en Estados Unidos donde el puntaje fluctuó entre un 48\% del ideal en 1970 y un 55\% el 2010 (27). Sin embargo, Norte Navarro y cols. (28), mostraron una situación más favorable, con un puntaje de $73,7 \pm 10,5$ en

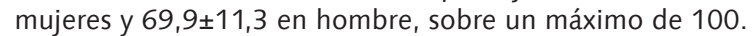

También existe consistencia en la mayoría de los estudios respecto a cuál son los alimentos críticos, con bajo consumo de frutas, verduras, lácteos, cereales integrales y alto consumo de alimentos procesados con alto contenido de grasas, azúcar y sodio (20-22).

TABLA 4

Índice de calidad de la alimentación según sexo, región, estado nutricional y edad.

\begin{tabular}{|c|c|c|c|c|c|}
\hline Variable & Número & $\begin{array}{c}\text { Poco saludable } \\
\%\end{array}$ & $\begin{array}{c}\text { Necesita Cambios } \\
\%\end{array}$ & $\begin{array}{c}\text { Saludable } \\
\%\end{array}$ & $\begin{array}{c}\text { Total } \\
\%\end{array}$ \\
\hline \multicolumn{6}{|l|}{ Sexo } \\
\hline Mujer & 5.430 & 35,1 & 55,1 & 9,8 & 100,0 \\
\hline \multirow[t]{2}{*}{ Hombre } & 4.005 & 36,0 & 55,5 & 8,5 & 100,0 \\
\hline & \multicolumn{4}{|c|}{$x 24,61 p$ NS } & \\
\hline \multicolumn{6}{|l|}{ Región } \\
\hline Norte & 2.075 & 30,3 & 60,5 & 9,2 & 100,0 \\
\hline Valparaíso & 2.291 & 32,6 & 53,2 & 14,2 & 100,0 \\
\hline Metropolitana & 4.085 & 35,0 & 54,5 & 10,4 & 100,0 \\
\hline \multirow[t]{2}{*}{ Sur } & 1.021 & 41,2 & 48,6 & 10,3 & 100,0 \\
\hline & \multicolumn{4}{|c|}{$x^{2} \quad 36,0 p<0,001$} & \\
\hline \multicolumn{6}{|c|}{ Estado nutricional } \\
\hline Bajo peso & 308 & 39,6 & 51,3 & 9,1 & 100,0 \\
\hline Normal & 6.508 & 34,4 & 56,0 & 9,6 & 100,0 \\
\hline Sobrepeso & 2.139 & 36,7 & 54,8 & 8,5 & 100,0 \\
\hline \multirow[t]{2}{*}{ Obeso } & 412 & 40,8 & 50,5 & 8,7 & 100,0 \\
\hline & \multicolumn{4}{|c|}{$x^{2} 13,0 p<0,05$} & \\
\hline \multicolumn{6}{|l|}{ Edad años } \\
\hline $17-19$ & 2.546 & 37,2 & 54,8 & 8,0 & 100,0 \\
\hline $25-24$ & 5.868 & 35,5 & 55,0 & 9,5 & 100,0 \\
\hline \multirow[t]{2}{*}{$25-29$} & 1.046 & 31,5 & 57,9 & 10,6 & 100,0 \\
\hline & \multicolumn{4}{|c|}{$x 215,4 p<0,005$} & \\
\hline Total & 9.460 & 35,4 & 55,3 & 9,3 & 100,0 \\
\hline
\end{tabular}

TABLA 5

Índice de calidad de la alimentación según año de la encuesta.

\begin{tabular}{|c|c|c|c|c|c|}
\hline Variable & Número & $\begin{array}{c}\text { Poco saludable } \\
\%\end{array}$ & $\begin{array}{c}\text { Necesita Cambios } \\
\%\end{array}$ & $\begin{array}{c}\text { Saludable } \\
\%\end{array}$ & $\begin{array}{c}\text { Total } \\
\%\end{array}$ \\
\hline \multicolumn{6}{|l|}{ Año } \\
\hline 2010 & 2.932 & 36,9 & 56,1 & 7,0 & 100,0 \\
\hline 2011 & 2.014 & 36,7 & 55,0 & 8,3 & 100,0 \\
\hline 2012 & 2.433 & 36,3 & 53,7 & 10,0 & 100,0 \\
\hline 2013 & 2.081 & 31,3 & 56,2 & 12,5 & 100,0 \\
\hline \multicolumn{6}{|c|}{$x^{2} 57,4 p<0,001$} \\
\hline
\end{tabular}


Es interesante destacar que existen pocas diferencias en la calidad de la alimentación según el estado nutricional, lo que refleja que es un problema transversal, que afecta en mayor o menor grado a toda la población. Diversos otros factores se han relacionados con la calidad del índice, entre los cuales destaca la edad, con una situación más negativa en los adolescentes, que en parte se relaciona con un estilo de vida menos estructurado, amplia jornada de estudios o laboral, dificultad en el acceso a alimentos saludables y factores económicos. Los factores económicos sin duda influyen no solo en los jóvenes, sino a toda edad, como lo refleja un estudio en EEUU (29).

Aun cuando la propuesta de este estudio no es original, tiene la ventaja de ser un instrumento de fácil aplicación y que puede ser adaptado a las recomendaciones de cada país. Otra fortaleza es que pudo ser aplicado en una muestra muy importante de estudiantes con representación del norte, centro y sur del país. Sus principales restricciones pueden derivar del hecho que el rango de edad en que fue aplicado es restringido y que los estudiantes universitarios y no representan exactamente los diversos niveles socio-económicos del país, variable que incide en la calidad de la dieta, por lo cual no es posible extrapolar los resultados a nivel nacional. Finalmente hubiera sido interesante evaluar la asociación entre la calidad de la dieta y el nivel de salud de la muestra estudiada, aunque ello representa un esfuerzo mayor.

Los resultados del estudio refuerzan la importancia de fortalecer a nivel nacional las actividades de promoción de una alimentación saludable, a través de todo el ciclo vital. Es difícil interpretar que factores influyeron en el cambio positivo en el índice observado en los últimos años, aunque este hecho sugiere que es posible modificar en parte las conductas. Es de esperar que el reglamento de la nueva ley de etiquetado nutricional, vigente desde junio del 2016, pueda contribuir a reducir el consumo de alimentos no saludables y a mejorar el puntaje a nivel nacional.

\section{RESUMEN}

Se han propuesto diversas metodologías para evaluar la calidad global de la alimentación, pero no existen índices específicos en Latinoamérica. El objetivo de este trabajo fue proponer un Índice de Alimentación Saludable de acuerdo a recomendaciones del Ministerio de Salud de Chile y validar su aplicación en una muestra nacional de estudiantes universitarios. Se utilizó la encuesta de frecuencia de consumo con 12 variables: 5 alimentos saludables, 4 no saludables y 3 de comidas principales. Cada variable fue evaluada de 1 (menos saludable) a 10 (recomendación ministerial) y el puntaje total se clasificó: saludable (90-120), necesita cambios (60-89) y poco saludable $(<60)$. Se encuestaron 9.452 estudiantes universitarios de diferentes y se evaluó el índice según sexo, edad y estado nutricional. La mediana fue solo 65,5 puntos (p25-75=54,5-77,9); 9,2\% saludable; 55,3\% necesitaba cambios y $35,5 \%$ poco saludable, sin diferencias por sexo y muy leves según estado nutricional $(p<0,05)$. A menor edad menos saludable $(p<0,005)$. El instrumento fue fácil de aplicar por profesionales de la salud y puede ser adaptado a las recomendaciones de cada país. Existen graves deficiencias en la calidad de la alimentación en estudiantes universitarios, independientemente de su estado nutricional y sexo.

Palabras claves: Índice; alimentación; saludable; estudiantes; Chile.

\section{REFERENCIAS}

1. World Health Organization. Diet, nutrition and the pre- vention of chronic diseases. Report of a Joint WHO/FAO Expert Consultation. WHO Technical Report Series no. 916, WHO, Geneva, 2013.

2. Nutrition and cancer. What science teaches us? González CA, Agudo A, Atalah E, López-Carrillo L, Navarro A, Editores. Editorial Médica Panamericana, Barcelona, España, 2015.

3. Choi JE, Ainsworth BE. Associations of food consumption, serum vitamins and metabolic syndrome risk with physical activity level in middle-aged adults: the National Health and Nutrition Examination Survey (NHANES) 2005-2006. Public Health Nutr. 2016; 19 (9):1674-83.

4. Wengreen $H$, Munger RG, Cutler $A$, et al. Prospective study of Dietary Approaches to Stop Hypertension- and Mediterranean-style dietary patterns and age-related cognitive change: the Cache County Study on Memory, Health and Aging. Am J Clin Nutr. 2013; 98:1263-71.

5. Heuer T, Krems C, Moon K, Brombach C, Hoffmann I. Food consumption of adults in Germany: results of the German National Nutrition Survey II based on diet history interviews. Br J Nutr. 2015; 113(10):1603-14.

6. Freire $W B$, Belmont $P$, López-Cevallos DF, Waters WF. ECuador's National Health and Nutrition Survey: objectives, design, and methods. Ann Epidemiol. 2015;25(11):877-8.

7. Mann KD, Pearce MS, McKevith B, Thielecke F, Seal CJ. Low whole grain intake in the UK: results from the National Diet and Nutrition Survey rolling programme 2008-11. Br J Nutr. 2015; 113(10):1643-51.

8. Atalah $E$, Amigo H, Bustos P. Does Chile's nutritional situation constitute a double burden? Am J Clin Nutr. 2014; 100(6):1623S-7S.

9. Gil Á, Martinez de Victoria E, Olza J. Indicators for the evaluation of diet quality. Nutr Hosp. 2015; 31(Suppl 3):128-44

10. Fransen HP, Ocké MC. Indices of diet quality. Curr Opin Clin Nutr Metab Care. 2008; 11:559-65.

11. Waijers PM1, Feskens EJ, Ocké MC. A critical review of predefined diet quality scores. Br J Nutr. 2007; 97:219-31.

12. Arvaniti F, Panagiotakos DB. Healthy indexes in public health practice and research: a review. Crit Rev Food Sci Nutr. 2008; 48:317-27.

13. Alkerwi A. Diet quality concept. Nutrition. 2014;30:613-8.

14. Fulgoni $V L$, Keast DR, Drewnowski A. Development and Validation of the Nutrient-Rich Foods Index: A Tool to Measure Nutritional Quality of Foods. J Nutr. 2009; 139:1549-54.

15. Kennedy ET, Oh/s J, Carlson S, Fleming $K$. The Healthy Eating Index: design and applications. J Am Diet Assoc. 1995; 95:1103-8.

16. Patterson RE, Haines PS, Popkin BM. Diet quality index: capturing a multidimensional behavior. J Am Diet Assoc. 1994; 94:57-64.

17. Huijbregts $P$, Feskens $E$, Rasanen L, Fidanza $F$, Nissinen A, Menotti A, Kromhout D. Dietary pattern and 20 year mortality in elderly men in Finland, Italy, and The Netherlands: longitudinal cohort study. BMJ. 1997; 315:13-7.

18. Trichopoulou A, Kouris-Blazos A, Wahlqvist ML, Gnardellis C, Lagiou P, Polychronopoulos E et al. Diet and overall survival in elderly people. BMJ. 1995; 311:1457-60.

19. Sotos-Prieto $M$, Moreno-Franco B, Ordovás JM, León $M$, Casasnovas JA, Peñalvo JL. Design and development of an instrument to measure overall lifestyle habits for epidemiological research: the Mediterranean Lifestyle (MEDLIFE) index. Public Health Nutr. 2015; 18(6):959-67. 
20. Ministry of Health. Pontifical Catholic University, Alberto Hurtado University. National Health Survey 2009-2010 ENS Chile. Available in: http://web.minsal.cl /portal/url/ item/bcb03d7bc28b64dfe040010165012d23.pdf, Consulted May 24, 2016.

21. Faculty of Medicine, Faculty of Economics and Business, University of Chile. National Food Consumption Survey. Final report. Available in: http://web.minsal.cl/sites/ default/files/ENCA-INFORME_FINAL.pdf. Consulted May 24, 2016.

22. Ratner $R$, Hernández $P$, Martel J, Atalah E. Food quality and nutritional status in university students of eleven Chilean regions. Rev Med Chil. 2012; 140:1571-79.

23. Pinheiro AC, Atalah E. Proposal of a method to assess global quality of diet. Rev Med Chil. 2005; 133:175-82.

24. Pereyra GI, Erazo M. Evaluation of the diet of women attended by the Chilean Public Health System. Arch Latinoam Nutr. 201; 61(3):254-61
25. Muñoz-Cano JM, Córdova-Hernández JA, Del ValleLeveaga $D$. The healthy eating index of new students at a university of Mexico. Nutr Hosp. 2015; 31(4):1582-8.

26. Closs VE, Pandolfo Feoli AM, Gomes I, Augustin Schwanke $\mathrm{CH}$. Healthy eating index of elderly: description and association with energy, macronutrients and micronutrients intake. Arch Latinoam Nutr. 2014; 64(1):34-41.

27. Miller PE, Reedy J, Kirkpatrick SI, Krebs-Smith SM. The United States food supply is not consistent with dietary guidance: evidence from an evaluation using the Healthy Eating Index-2010. J Acad Nutr Diet. 2015; 115(1):95-100

28. Norte Navarro Al, Ortiz Moncada R. Spanish diet quality according to the healthy eating index. Nutr Hosp. 2011; 26(2):330-6.

29. Rehm CD, Monsivais $P$, Drewnowski A. Relation between diet cost and Healthy Eating Index 2010 scores among adults in the United States 2007-2010. Prev Med. 2015; 73:70-5. 